\title{
Transepithelial electrical resistance (TEER): a functional parameter to monitor the quality of oviduct epithelial cells cultured on filter supports
}

\author{
Shuai Chen ${ }^{1,2} \cdot$ Ralf Einspanier $^{3} \cdot$ Jennifer Schoen $^{2}$
}

Accepted: 4 July 2015 / Published online: 31 July 2015

(C) The Author(s) 2015. This article is published with open access at Springerlink.com

\begin{abstract}
Cultivation of oviduct epithelial cells on porous filters fosters in vivo-like morphology and functionality. However, due to the optical properties of the filter materials and the cells' columnar shape, cell quality is hard to assess via light microscopy. In this study, we aim to evaluate transepithelial electrical resistance (TEER) measurement as a prognostic quality indicator for the cultivation of porcine oviduct epithelial cells (POEC). POEC were maintained in four different types of media for 3 and $6 \mathrm{w}$ to achieve diverse culture qualities, and TEER was measured before processing samples for histology. Culture quality was scored using morphological criteria (presence of cilia, confluence and cell polarity). We furthermore analyzed the correlation between cellular height (as a measure of apical-basal polarization) and TEER in fully differentiated routine cultures (biological variation) and in cultures with altered cellular height due to hormonal stimulation. Fully differentiated cultures possessed a moderate TEER between 500 and $1100 \Omega * \mathrm{~cm}^{2}$. Only $5 \%$ of cultures which exhibited TEER values in this defined range had poor
\end{abstract}

Electronic supplementary material The online version of this article (doi:10.1007/s00418-015-1351-1) contains supplementary material, which is available to authorized users.

Jennifer Schoen

schoen.jennifer@fbn-dummerstorf.de

1 College of Life Science, Hebei University, Baoding 071002, Hebei, People's Republic of China

2 Institute of Reproductive Biology, Leibniz Institute for Farm Animal Biology, Wilhelm-Stahl-Allee 2, 18196 Dummerstorf, Germany

3 Institute of Veterinary Biochemistry, Department of Veterinary Medicine, Freie Universität Berlin, Oertzenweg 19b, 14163 Berlin, Germany quality. Sub-differentiated cultures showed either very low or excessively high TEER. We unveiled a highly significant $(P<0.0001)$ negative linear correlation between TEER and epithelial height in well-differentiated cultures (both routine and hormone stimulated group). This may point toward the interaction between tight junction assembly and epithelial apical-basal polarization. In conclusion, TEER is a straightforward quality indicator which could be routinely used to monitor the differentiation status of oviduct epithelial cells in vitro.

Keywords TEER - Oviduct epithelial cells · Culture quality $\cdot$ Tight junctions

\section{Introduction}

Culturing epithelial cells on porous filter supports mimics in vivo-like conditions by allowing nutrient supply from the basolateral compartment and therefore promotes cell differentiation and closer (morphological as well as functional) similarity to the native tissue (Zegers et al. 2003). Transepithelial electrical resistance (TEER) measurement is used to assess the barrier function of epithelial cells on these porous supports. When assessing the electrical impedance, a continuous current passes through the cells on both transcellular and paracellular paths (Powell 1981). The transcellular resistance is primarily made up by the apical and basolateral plasma membrane, while the paracellular resistance is a result of cell-substrate as well as cell-cell contacts. It is known that specific tight junction proteins largely influence epithelial resistance (Anderson 2001; Lo et al. 1999). Hence, the TEER value reflects physical structures and properties of filter-grown epithelial cultures. So far, TEER measurement has been mainly 
applied for assessing the permeability of tight junctions or the membrane perturbation by toxicants on intestinal and kidney epithelial cell lines (Narai et al. 1997; Velarde et al. 1999; Madgula et al. 2007).

The oviduct epithelium is the reproductive venue where transport and maturation of gametes, fertilization and cleavage-stage embryonic development occur (Coy et al. 2012). Taking advantage of porous filter supports, recently primary oviduct epithelial cultures with improved cell differentiation have been generated from species including: monkey, human, pig and cattle (Chen et al. 2013b; Levanon et al. 2010; Palma-Vera et al. 2014; Rajagopal et al. 2006; Gualtieri et al. 2012). Tools allowing non-invasive quality control are highly desirable for these differentiated models as filter-supported techniques are more complex compared to basic cell cultures. Moreover, these models are normally maintained over prolonged time periods (up to $6 \mathrm{w}$ ), which further necessitates quality monitoring. There are two major factors interfering with routine morphological quality control via light microscopy: (1) The commercial membrane materials are polycarbonate $(\mathrm{PC})$ or polyester (PET) with a thickness $\geq 10 \mu \mathrm{m}$; (2) cells differentiate in the apical-basal axis reaching a height up to $18 \mu \mathrm{m}$ (Chen et al. 2013b). The polarization status in the apical-basal dimension (columnar shape) is therefore hard to assess by microscopy of living cells, although it is a crucial quality criterion in this cell type.

Recently, we validated a standardized culture model of porcine oviduct epithelial cells (POEC) in large-scale trials, which exhibits distinct hormonal responsiveness during estrous cycle simulation (Chen et al. 2013a, b). In this follow-up study, we evaluate whether TEER measurement could serve as a straightforward quality indicator for differentiated oviduct epithelial models. To obtain cultures with diverse qualities, cells were maintained in four different types of media for 3 and $6 \mathrm{w}$. We examined the association between TEER values and the morphological features of cultured cells, and further classified TEER values into ranges for quality indication. We also unveiled how TEER values correlate with apical-basal polarization (measured as cellular height) in routinely cultured as well as hormonally stimulated cells.

\section{Materials and methods}

\section{Materials}

If not otherwise indicated, all cell culture reagents and materials (including 24-well PET Millicell inserts PIHT12R48 and fetal bovine serum FBS S0115) were acquired from Merck Millipore. Chemicals for histological procedures were obtained from Carl Roth. EVOM2
Epithelial Voltohmmeter together with STX2 electrode was purchased from World Precision Instruments (WPI).

\section{Tissue samples and cell culture}

Oviducts were collected from 6-month-old gilts (Large White $\times$ German Landrace) in the local slaughterhouse immediately after slaughter. Cell isolation and filter-supported cultivation were performed following the protocol previously reported by our group (Miessen et al. 2011; Chen et al. 2013b). Cell cultures with divergent qualities were generated by using four types of media: (M1) Ham's F12 with $10 \%$ charcoal-stripped FBS (S181F, Biowest); (M2) two parts of M1 plus one part of the correspondent 3T3-conditioned medium; (M3) Ham's F12 with $10 \%$ FBS (S0115, Biochrom); and (M4) two parts of M3 plus one part of the corresponding 3T3-conditioned medium. All media were supplemented with $100 \mathrm{U} / \mathrm{mL}$ penicillin, $100 \mu \mathrm{g} / \mathrm{mL}$ streptomycin, $50 \mu \mathrm{g} / \mathrm{mL}$ gentamicin, $1 \mu \mathrm{g} / \mathrm{mL}$ amphotericin $\mathrm{B}, 10 \mathrm{mg} / \mathrm{mL}$ reduced glutathione and $10 \mathrm{mg} /$ $\mathrm{mL}$ ascorbic acid (Sigma). 3T3-conditioned medium was prepared following the standard protocol (Freshney 2005). Air/liquid culture was achieved by removing the medium $(200 \mu \mathrm{l})$ in the apical compartment $48 \mathrm{~h}$ after seeding.

\section{Morphology and morphometric assessment}

To harvest cells, membranes were cut out from inserts and washed in PBS followed by $2 \mathrm{~h}$ fixation in Bouin's solution. Subsequently, membranes were cut in half, vertically settled in $2 \%$ agarose and post-fixed in $4 \%$ formalin. After dehydration in ascending ethanol series, samples were embedded in paraplast. Approximately, 3- $\mu$ m-thick sections were prepared from the middle part of the membrane. Finally, slides were stained with hematoxylin/eosin (HE) and analyzed by light microscopy.

Cultures grown in different media were scored using qualitative morphological criteria including: presence of cilia, cell polarity (columnar shape) and confluence of the culture (Table 1). Scores for "cilia" and "polarity" were assigned ranging from 0 to 3, while scores for "confluence" were assigned ranging from 0 to 2 . POEC were cultured for $3 \mathrm{w}(N=4$ animals $)$ and $6 \mathrm{w}(N=6$ animals $)$ in all four types of media, respectively. Detailed scores for each criterion and relevant TEER values are listed in the supplementary table (S1).

To assess the correlation between TEER and apicalbasal polarization of the cell culture, we further selected cellular height as a quantitative morphometric parameter. As all cultures in M4 achieved highest quality, M4 was used for this part of the study. Histomorphometric height measurements were taken in (1) routine cell cultures: cells grown for $3 \mathrm{w}(N=23$ animals) and $6 \mathrm{w}(N=23$ animals $)$; 
Table 1 Evaluation criteria for morphological scoring of POEC

\begin{tabular}{llll}
\hline Score & Cilia & Polarity & Confluence \\
\hline 3 & Dense cilia & Cellular height $\geq 10 \mu \mathrm{m}$ & $/$ \\
2 & Cilia in certain areas & $5 \mu \mathrm{m}<$ cellular height $<10 \mu \mathrm{m}$ & Fully confluent \\
1 & Very few cells with cilia & $2 \mu \mathrm{m}<$ cellular height $<5 \mu \mathrm{m}$ & Partly confluent \\
0 & No cilia & No cellular polarity visible & No or few cells on membrane \\
\hline
\end{tabular}

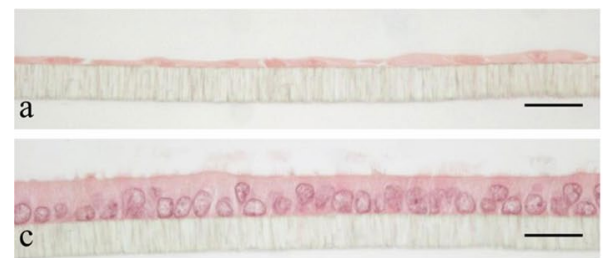

Fig. $1 \mathrm{HE}$ staining of primary porcine oviduct epithelial cells (POEC) cultured in media M1 (a), M2 (b), M3 (c) and M4 (d), respectively, for $6 \mathrm{w}$. M1: Ham's F12 + $10 \%$ charcoal-stripped FBS; M2: M1 enriched with corresponding 3T3-conditioned medium at a

2) hormonal stimulation experiment: cells treated with $35 \mathrm{ng} / \mathrm{ml}$ progesterone (P4; Sigma-Aldrich) and $10 \mathrm{pg} / \mathrm{mL}$ $17 \beta$-estradiol (E2; Sigma-Aldrich) for the last 10 days of the $3 \mathrm{w}(N=11$ animals $)$ and $6 \mathrm{w}(N=11$ animals $)$ culture period. As we reported earlier, this stimulation protocol changes the functional characteristics of the oviduct epithelial cells and allows simulation of the changes observed during the estrous cycle in vivo (Chen et al. 2013a). Controls (cells treated with ethanol solvent only) were included in the hormonal stimulation experiment. A total of 15 histomorphometric height measurements were taken for each sample following a recently published protocol (Chen et al. 2013a).

\section{TEER measurement}

We routinely performed TEER measurements for all samples shortly before fixation. To eliminate the influence of temperature, measures were performed within $5 \mathrm{~min}$ after taking the culture plates out of the incubator. Within this time, samples did not show any reading drift. Before measurement, electrodes were equilibrated and sterilized according to the manufacturer's recommendations. Two hundred microliters of culture medium was added in the upper compartment of the cell culture system. The ohmic resistance of a blank (culture insert without cells) was measured in parallel. To obtain the sample resistance, the blank value was subtracted from the total resistance of the sample. The final unit area resistance $\left(\Omega^{*} \mathrm{~cm}^{2}\right)$ was calculated by multiplying the sample resistance by the effective area of the membrane $\left(0.33 \mathrm{~cm}^{2}\right.$ for 24 -well Millicell inserts).

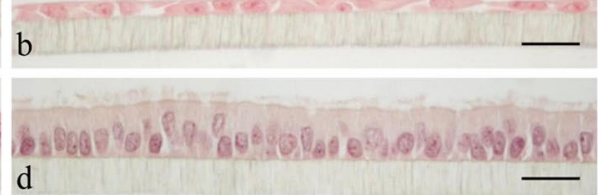

ratio of 2:1(V: V); M3: Ham's F12 + $10 \%$ FBS; M4: M3 enriched with corresponding 3T3-conditioned medium at a ratio of $2: 1(\mathrm{~V}: \mathrm{V})$; magnification $\times 400$, scale bars $20 \mu \mathrm{m}$

\section{Statistics}

Statistical data were analyzed by SPSS Statistics 20 for Windows. Correlation coefficient was assessed by Pearson test, while linear regression analyses were used to determine the slope between TEER values and cellular heights. A $P$ value $<0.01$ was considered statistically significant.

\section{Results}

\section{TEER and morphological quality of POEC}

Cells exhibited disparate architectures when subjected to different media. Overall, both charcoal-stripped media lead to poor morphological quality: Cells were dedifferentiated, flat or dwarf, lost cilia and secretory protrusions (Fig. 1a, b), with an average morphology score of 2.6 in M1 and 4.2 in M2 (Fig. 2a, b). Their correspondent TEER values varied tremendously from 0 to $2270 \Omega * \mathrm{~cm}^{2}$. In contrast, 9 out of 10 cultures in M3, and all cultures in M4 achieved highest quality (score $=8$ ). Cells were highly polarized, confluent and retained a mix of ciliated and secretory cells (Fig. 1c, d). They displayed consistent and moderate TEER readings from 729 to $1070 \Omega * \mathrm{~cm}^{2}$ (Fig. 2c, d). The best culture (score $=7$ ) in M2 also showed a medium TEER output (Fig. 2b); the only outlier in M3, which had a low morphology score of 5 (confluence was given, see S1), exhibited a low TEER reading (Fig. 2c).

To better visualize the relation between TEER and culture quality, we pooled data from above four media and divided them into two groups: good quality (score $\geq 7$ ) 

and corresponding TEER values of POEC after $3 \mathrm{w}(N=4$ animals) and $6 \mathrm{w}(N=6$ animals $)$ cultivation. Cells are grown in media M1 (a), M2 (b), M3 (c) and M4 (d), respectively. Left $y$ axis: morphological scores; right $y$ axis: TEER values $\left(\Omega * \mathrm{~cm}^{2}\right)$. Shadow area in $(\mathbf{b})$ highlights a well-differentiated sample with moderate TEER value; shadow area in (c) highlights a poorly differentiated sample with low TEER value
Fig. 2 Morphological scores
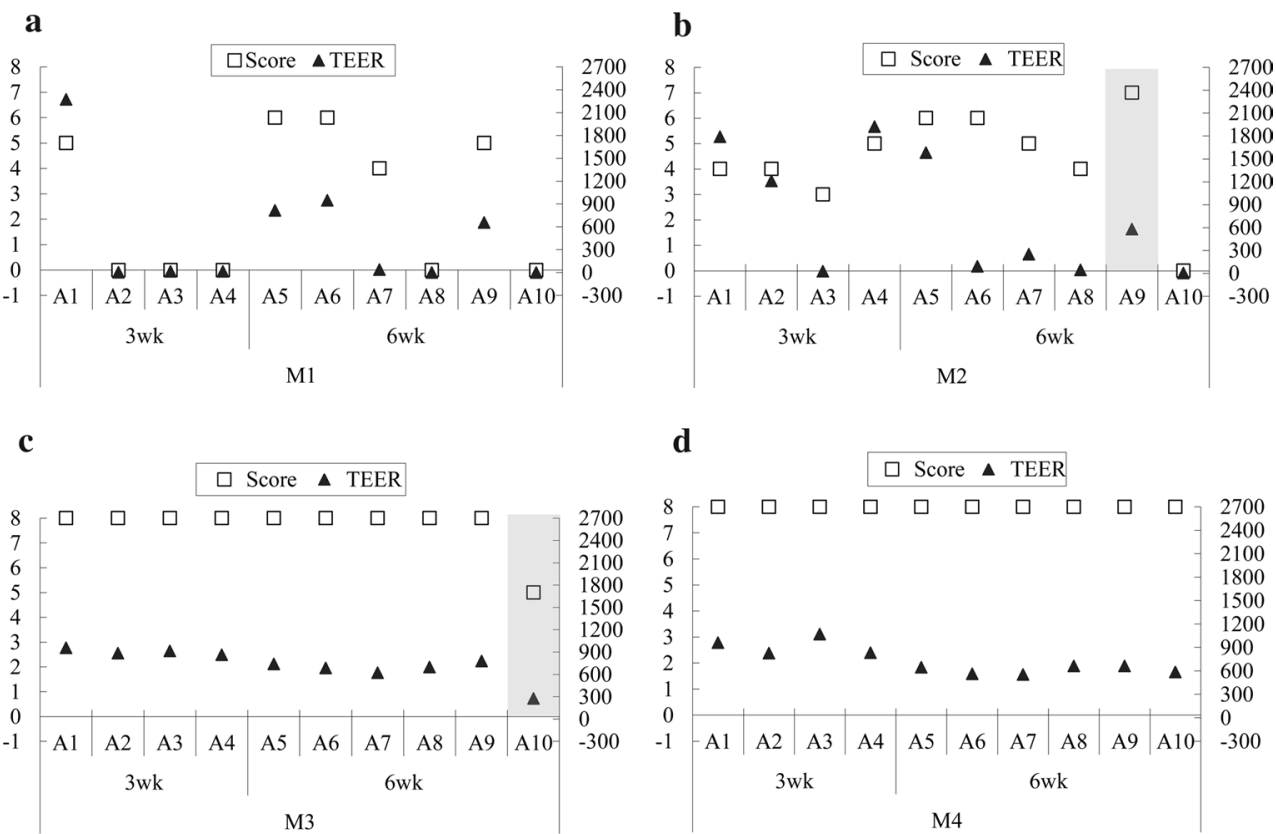

Fig. 3 TEER in POEC cultures with divergent quality and with or without hormonal stimulation. a TEER of welldifferentiated (score $\geq 7$ ) and non-differentiated (score $<7$ ) POEC cultures. $\mathbf{d}$ TEER of POEC cultures in the hormonal stimulation experiment. Treated group: Cells are first cultured for 11 or 32 days and then treated with $35 \mathrm{ng} / \mathrm{ml} \mathrm{P} 4$ and $10 \mathrm{pg} / \mathrm{ml} \mathrm{E} 2$ for 10 days; control group: cells received solvent only. b Representative histological picture of a POEC culture with high TEER $\left(1579 \Omega * \mathrm{~cm}^{2}\right)$. c POEC culture with low TEER $\left(25.25 \Omega * \mathrm{~cm}^{2}\right)$. e POEC culture under hormonal stimulation (P4 dominance) with a TEER of $840 \Omega^{*} \mathrm{~cm}^{2}$. f Control POEC in the hormonal stimulation experiment (solvent only; TEER of $724 \Omega * \mathrm{~cm}^{2}$ ). (b, c, e, f) HE staining, magnification $\times 400$, scale bars $20 \mu \mathrm{m}$

\section{Summerized TEER ( all 4 media)}

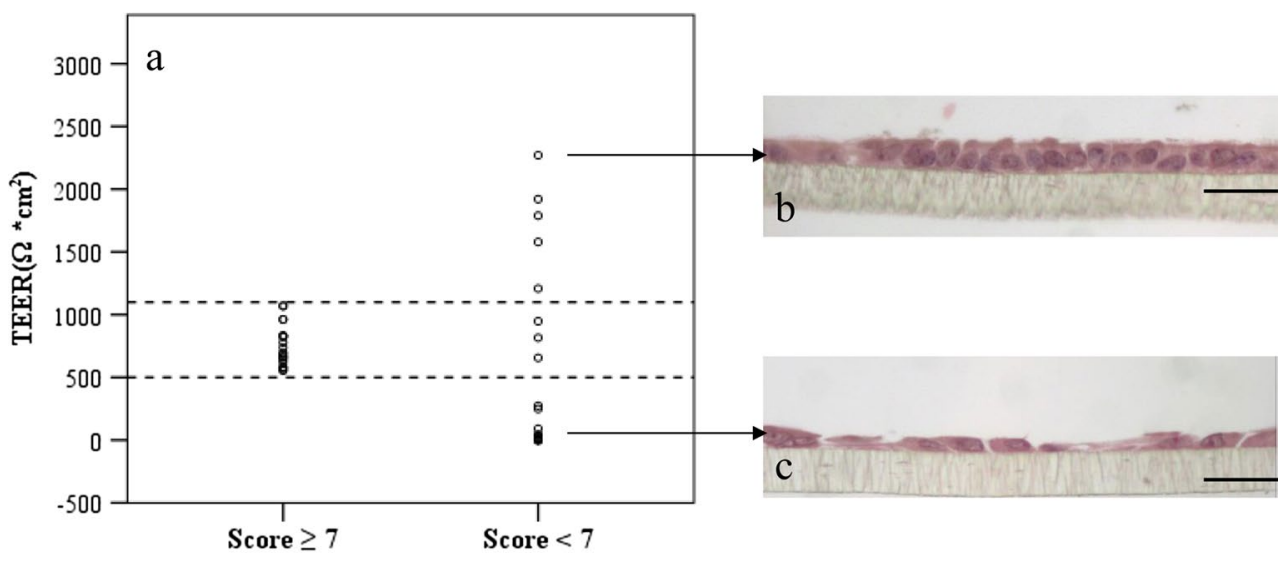

Hormonal stimulation

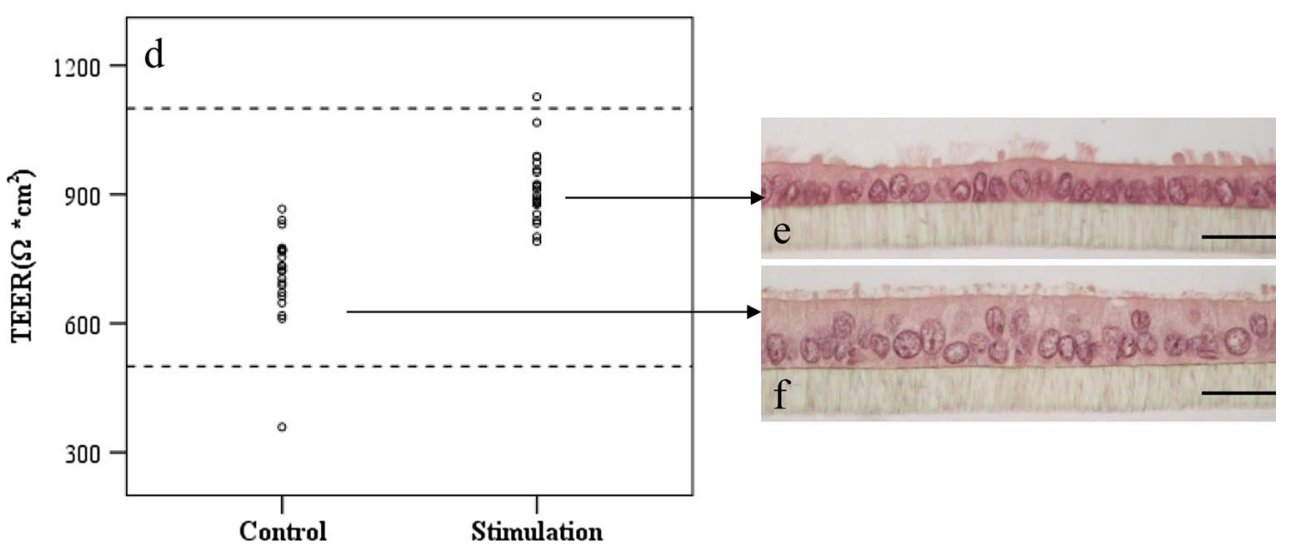

and poor quality group (score < 7). TEER of all morphological intact cultures fell into a tight range from 500 to $1100 \Omega * \mathrm{~cm}^{2}$, while $85 \%$ of cultures with low morphological quality possessed resistance either exceeding $1100 \Omega^{*} \mathrm{~cm}^{2}$ or below $500 \Omega^{*} \mathrm{~cm}^{2}$ (Fig. 3a). Histological investigation further revealed that partway differentiated 
Fig. 4 Negative correlation between TEER and cellular height. a POEC grown in M4 for $3 \mathrm{w}$ ( $N=23$ animals) and 6w $(N=23$ animals; $n$ total samples $=46 ; R=-0.569$, $P<0.0001)$. b Hormonal stimulation experiment $(n$ total samples $=44)$. Cells $(N=11$ animals) are subjected to hormonal or solvent treatment during either $3 \mathrm{w}$ or $6 \mathrm{w}$ of culture in M4 $(R=-0.852, P<0.00001)$



epithelial layers tend to yield over high TEER output (Fig. 3b). Contrarily, completely undifferentiated cultures (no visible polarization) had extremely low TEER values (Fig. 3c). Similarly, in the hormonal stimulation experiment $>95 \%$ of the cultures exhibited the proposed TEER range. All of them demonstrated fully differentiated morphology (Fig. 3d). The hormonally stimulated group (P4 domination) tends to possess higher TEER outputs but lower cellular height (Fig. 3e, f).

\section{TEER negatively correlates with cellular height}

As all cultures tested in M4 were well differentiated, we selected medium M4 for further analysis. When we expanded experimental trials up to 46 in total $(N=23$ animals, cultured for $3 \mathrm{w}$ and $6 \mathrm{w}$, respectively), all TEER values turned out to fit in our proposed TEER range (Fig. 4a). Furthermore, a significant $(R=-0.569, P<0.0001)$ negative linear correlation was observed between TEER and cellular height, with the regression equation of $Y=27.132-0.017 \mathrm{X}$.

We also analyzed the correlation between these two parameters in the hormonal stimulation experiment. Again, TEER values revealed similar negative correlation with cellular height $(R=-0.852, P<0.00001$, Fig. $4 \mathrm{~b})$ with a linear regression equation of $Y=29.840-0.021 \mathrm{X}$.

\section{Discussion}

The recent advance of filter-supported oviductal epithelial cultures, which proved closer tissue-like morphology and biology than conventional cultures, necessitates a suitable monitoring technique to assess their culture qualities. After systematic analysis of TEER measurement in conjunction with morphological features of POEC achieved in varied types of media, we clearly revealed how the electrical impedance associates with differentiation status of epithelial cultures. Furthermore, we may conclude a defined TEER range for high-quality cultures $\left(500-1100 \Omega * \mathrm{~cm}^{2}\right.$ in 24-well PET inserts, $0.4 \mu \mathrm{m}$ pore size), which could be used as guidance for the cultivation of oviduct epithelial cells on filter supports. In the study presented here, $90 \%$ of cultures with deprived morphology had TEER values below 500 or above $1100 \Omega * \mathrm{~cm}^{2}$. In contrary, only $5 \%$ of cultures which exhibited TEER values between 500 and $1100 \Omega * \mathrm{~cm}^{2}$ had poor quality.

When considering the epithelial layer as a simple electrical structure, it could be described as two parallel units: a transcellular unit and a paracellular unit which is influenced mainly by tight junctions, as well as intercellular spaces and cell-substrate contacts (Lo et al. 1999; Powell 1981). According to the permeability of epithelial tissues, they could be classified into two categories: leaky and tight epithelia. Epithelia are considered leaky, when their TEER is below $1000 \Omega * \mathrm{~cm}^{2}$, with a paracellular conductance greater than transcellular conductance (Powell 1981). Oviduct epithelium is described to be leaky (Leese 1988); therefore, in our POEC cultures, the TEER measurement largely reflected the changes in paracellular unit, mainly the elaboration of strands of tight junctions (Cereijido et al. 2008; Anderson 2001; Claude 1978; Claude and Goodenough 1973).

It is widely assumed that low TEER indicates low quality of cultured epithelial cells; however, here we clearly demonstrate that excessive transepithelial resistance in cells originating from a leaky epithelium is associated with a sub-differentiated culture status.

The proposed TEER range to forecast the quality of oviductal epithelia in vitro is further validated by comparison with well-differentiated cultures from other species: The primary culture of monkey oviduct epithelium, which closely resembled native tissue, exhibited a TEER of $750 \Omega * \mathrm{~cm}^{2}$ by day 20 of culture (Rajagopal et al. 2006). When cultured for $17 \mathrm{~d}$, oviductal epithelial cells from rabbits at estrous showed a TEER of $851 \Omega * \mathrm{~cm}^{2}$ (Edwards and 
Leese 1993). As in our experiment, both studies employed the $\mathrm{Ag} / \mathrm{AgCl}$ electrode method for their resistance measurement. Investigations applying Ussing chambers produced relatively lower TEER values between 150 and $234 \Omega * \mathrm{~cm}^{2}$ (Mahmood et al. 2001; Rajagopal et al. 2006). A study comparing both methods on the same cell type and culture system found around threefold lower TEER values when using the Ussing chamber (Rajagopal et al. 2006). This may be explained due to the different geometries of the two devices, leading to discrepancies in the current path/path lengths cross through the membrane, hence yielding divergent resistance outputs.

Apart from the inherent characteristics of epithelial cells, other factors also affect the transepithelial impedance. It has been shown that material and physical properties of the membrane support could affect cell morphology and cellsubstrate contacts, therefore leading to divergent TEER values (Lo et al. 1999; Thwaites et al. 1993). The results we generated in this study are all based on PET $(0.4 \mu \mathrm{m}$ pore size) membranes. Besides, previous studies indicated that temperature has strong influence on the TEER measurement and may skew results (Matter and Balda 2003).

Finally, taking advantage of our standardized POEC model, we were able to quantify the cell polarization along the apical-basal axis and link it to electrical impedance assessment using TEER. To our knowledge, we could demonstrate for the first time a fine-tuned inversed correlation between cellular height, a parameter reflecting the degree of apical-basal polarity and TEER which mainly corresponds to the strength of cellular junctions.

It has been reported that in epithelial tissues, many tight junction complexes (e.g., CRB3, Par) are involved in the polarization process (Shin et al. 2006; Etienne-Manneville and Hall 2003). However, the underlying molecular mechanisms which co-regulate these two events need to be clarified in further studies. The mathematical equation we provided may facilitate the prediction of porcine oviductal epithelial cell height based on a known TEER value.

To conclude, TEER measurement is a non-invasive tool for the quality assessment of filter-based oviduct epithelial cultures. We hereby presented a proper TEER range which could be used to predict the apical-basal polarization in these cultures. Furthermore, we firstly unveiled the inverse relationship between epithelial height and impedance, which might promote our understanding of the interconnection between apical and basal polarity and tight junction assembly.

Acknowledgments We thank the Vion Lausitz GmbH, Kasel-Golzig, Germany, for providing porcine oviduct tissue. This work was supported by the Federal Institute for Risk Assessment (BfR, FK 1329-473), Germany; SC was supported by the Nature Science Funds from Hebei Education Ministry (QN2014124).

\section{Compliance with ethical standards}

Conflict of interest The authors declare that no conflict of interest exists.

Open Access This article is distributed under the terms of the Creative Commons Attribution 4.0 International License (http://creativecommons.org/licenses/by/4.0/), which permits unrestricted use, distribution, and reproduction in any medium, provided you give appropriate credit to the original author(s) and the source, provide a link to the Creative Commons license, and indicate if changes were made.

\section{References}

Anderson JM (2001) Molecular structure of tight junctions and their role in epithelial transport. News Physiol Sci 16:126-130

Cereijido M, Contreras RG, Shoshani L, Flores-Benitez D, Larre I (2008) Tight junction and polarity interaction in the transporting epithelial phenotype. Biochim Biophys Acta 1778(3):770-793

Chen S, Einspanier R, Schoen J (2013a) In vitro mimicking of estrous cycle stages in porcine oviduct epithelium cells: estradiol and progesterone regulate differentiation, gene expression, and cellular function. Biol Reprod 89(3):54

Chen S, Einspanier R, Schoen J (2013b) Long-term culture of primary porcine oviduct epithelial cells: validation of a comprehensive in vitro model for reproductive science. Theriogenology 80(8):862-869

Claude P (1978) Morphological factors influencing transepithelial permeability: a model for the resistance of the zonula occludens. J Membr Biol 39(2-3):219-232

Claude P, Goodenough DA (1973) Fracture faces of zonulae occludentes from "tight" and "leaky" epithelia. J Cell Biol 58(2):390-400

Coy P, García-Vázquez FA, Visconti PE, Avilés M (2012) Roles of the oviduct in mammalian fertilization. Reproduction 144(6):649-660

Edwards LJ, Leese HJ (1993) Glucose transport and metabolism in rabbit oviduct epithelial cells. J Reprod Fertil 99(2):585-591

Etienne-Manneville S, Hall A (2003) Cell polarity: Par6, aPKC and cytoskeletal crosstalk. Curr Opin Cell Biol 15(1):67-72

Freshney RI (Chihester : John Wiley [distributor], 2005) Culture of animal cells. A manual of basic technique/R. Ian Freshney. Wiley-Liss, Hoboken, N.J

Gualtieri R, Mollo V, Braun S, Barbato V, Fiorentino I, Talevi R (2012) Long-term viability and differentiation of bovine oviductal monolayers: bidimensional versus three-dimensional culture. Theriogenology 78(7):1456-1464

Leese HJ (1988) The formation and function of oviduct fluid. J Reprod Fertil 82(2):843-856

Levanon K, Ng V, Piao HY, Zhang Y, Chang MC, Roh MH, Kindelberger DW, Hirsch MS, Crum CP, Marto JA, Drapkin R (2010) Primary ex vivo cultures of human fallopian tube epithelium as a model for serous ovarian carcinogenesis. Oncogene 29(8):1103-1113

Lo CM, Keese CR, Giaever I (1999) Cell-substrate contact: another factor may influence transepithelial electrical resistance of cell layers cultured on permeable filters. Exp Cell Res 250(2):576-580

Madgula VL, Avula B, Reddy VL, Niranjan Khan IA, Khan SI (2007) Transport of decursin and decursinol angelate across Caco-2 and MDR-MDCK cell monolayers: in vitro models for intestinal and blood-brain barrier permeability. Planta Med 73(4):330-335 
Mahmood T, Djahanbakhch O, Burleigh D, Puddefoot JR, Vinson GP (2001) The effect of cAMP on ion transport in Fallopian tube epithelial cells in vitro. Mol Hum Reprod 7(10):957-961

Matter K, Balda MS (2003) Functional analysis of tight junctions. Methods 30(3):228-234

Miessen K, Sharbati S, Einspanier R, Schoen J (2011) Modelling the porcine oviduct epithelium: a polarized in vitro system suitable for long-term cultivation. Theriogenology 76(5):900-910

Narai A, Arai S, Shimizu M (1997) Rapid decrease in transepithelial electrical resistance of human intestinal Caco-2 cell monolayers by cytotoxic membrane perturbents. Toxicol In Vitro 11(4):347-354

Palma-Vera S, Einspanier R, Schoen J (2014) Bovine oviductal epithelial cells: long term culture characterization and impact of insulin on cell morphology. Reprod Biol 14(3):206-212

Powell DW (1981) Barrier function of epithelia. Am J Physiol 241(4):G275-G288
Rajagopal M, Tollner TL, Finkbeiner WE, Cherr GN, Widdicombe JH (2006) Differentiated structure and function of primary cultures of monkey oviductal epithelium. In Vitro Cell Dev Biol Anim 42(8-9):248-254

Shin K, Fogg VC, Margolis B (2006) Tight junctions and cell polarity. Annu Rev Cell Dev Biol 22:207-235

Thwaites DT, Hirst BH, Simmons NL (1993) Passive transepithelial absorption of thyrotropin-releasing hormone (TRH) via a paracellular route in cultured intestinal and renal epithelial cell lines. Pharm Res 10(5):674-681

Velarde G, Ait-Aissa S, Gillet C, Rogerieux F, Lambre C, Vindimian E, Porcher JM (1999) Use of transepithelial electrical resistance in the study of pentachlorophenol toxicity. Toxicol In Vitro 13(4-5):723-727

Zegers MMP, O'Brien LE, Yu W, Datta A, Mostov KE (2003) Epithelial polarity and tubulogenesis in vitro. Trends Cell Biol 13(4):169-176 